\title{
Hormonal and Gynecological Risk Factors in Frontal Fibrosing Alopecia: A Case-Control Study
}

\author{
Diego Buendía-Castaño ${ }^{a}$ David Saceda-Corralo ${ }^{a}, b \quad$ Oscar M. Moreno-Arrones ${ }^{a}$ \\ Pablo Fonda-Pascual ${ }^{\mathrm{a}} \quad$ Adrián Alegre-Sánchez $^{\mathrm{a}} \quad$ Cristina Pindado-Ortega $^{\mathrm{a}}$ \\ Pablo Fernandez-Gonzalez ${ }^{a}$ Sergio Vañó-Galván ${ }^{a, b}$ \\ ${ }^{a}$ Dermatology Service, Trichology Unit, Ramon y Cajal Hospital, and b Trichology Unit, Grupo Pedro Jaén Clinic, \\ Madrid, Spain
}

\section{Keywords}

Frontal fibrosing alopecia $\cdot$ Hormonal background . Gynecological background · Menopause · Tamoxifen . Intrauterine device ly proposed hypothesis of an underlying hormonal mechanism in the etiopathogenesis of FFA and point out low-estrogen environments as an ideal condition forFFA development.

(c) 2017 S. Karger AG, Basel

\section{Introduction}

Frontal fibrosing alopecia (FFA) is a scarring alopecia with a yet unknown etiology. Familiar aggregation and an increasing incidence, respectively, suggest the implication of genetic and environmental factors in the development of the disease. Also, some observations, such as the predominance in postmenopausal women or the therapeutic usefulness of antiandrogenic drugs [1,2], suggest the coexistence of a hormonal mechanism. However, there is scarce evidence demonstrating the presence of hormonal background differences in FFA patients when compared to the general population. The objective of this study was to explore potential risk factors for FFA related to patients' lifetime gynecological and obstetric events and pathologies.

\section{Materials and Methods}

A single-center case-control study was designed, including female FFA patients diagnosed between 2014 and 2016. Diagnosis of FFA was made clinically (recession of the frontal hair line, perifollicular erythema, and loss of eyebrows) with histological confirma-

\section{KARGER}

() 2017 S. Karger AG, Basel 
Table 1. Quantitative variables with $U$ value of the Mann-Whitney U test

\begin{tabular}{llll}
\hline & Cases & Controls & Uvalue \\
\hline Age, years $(n=312)$ & $62.6(38-85)$ & $62.4(36-86)$ & $\mathrm{ns}$ \\
Age of menarche, years $(n=311)$ & $12.6(9-16)$ & $12.7(9-16)$ & $\mathrm{ns}$ \\
Age of menopause, years $(n=289)$ & $47.7(33-58)$ & $49.7(30-58)$ & 0.01 \\
Estimated fertile life, years $(n=289)$ & $34.8(21-49)$ & $36.5(15-46)$ & 0.004 \\
Number of pregnancies $(n=312)$ & $2.5(0-8)$ & $2.3(0-7)$ & $\mathrm{ns}$ \\
Age of first pregnancy $(n=289)$ & $27.5(18-43)$ & $27.0(17-42)$ & $\mathrm{ns}$ \\
Number of abortions $(n=312)$ & $0.4(0-4)$ & $0.3(0-4)$ & $\mathrm{ns}$ \\
Number of live births $(n=312)$ & $2.1(0-5)$ & $2.0(0-6)$ & $\mathrm{ns}$ \\
Total months of breastfeeding $(n=293)$ & $8.5(0-51)$ & $7.0(0-43)$ & $\mathrm{ns}$ \\
\hline
\end{tabular}

Values are means with ranges in parentheses. ns, not significant. ${ }^{a}$ Fertile life: calculated as age of menopause - age of menarche for postmenopausal women, and as age - age of menarche for premenopausal women. ${ }^{b}$ Breastfeeding was recorded in the questionnaire as months of breastfeeding for each child and total time of breastfeeding in months.

tion when needed. Two female controls were recruited for each case in our outpatient consultation area in the last trimester of 2016 and were matched with patients by groups encompassing 3 years of age. Patients with consultation reasons other than scarring alopecia and patients' companions were included as controls. Both cases and controls were interviewed, and extensive data about their gynecological and hormonal medical history were recorded. Variables recorded included age, age of menarche, age of menopause, and years of fertile life (calculated as the difference between age of menopause and age of menarche for postmenopausal women or as the difference between age and age of menarche for premenopausal women). Regarding fertility, we gathered data about the total number of pregnancies, the number of live births and abortions, the age of first pregnancy, use of oral contraceptives and time of consumption, use of intrauterine device (IUD) and time of use, and medical fertility treatments and assisted fertility treatments (artificial insemination and in vitro fecundation). Breastfeeding was recorded in the questionnaire as months of breastfeeding for each child and total time of breastfeeding in months. Personal history of hysterectomy and oophorectomy (unilateral or bilateral) as well as breast cancer and related treatments (tamoxifen and antiandrogens) were also recorded.

For quantitative variables, means and ranges were calculated and differences were assessed using the Mann-Whitney $U$ test. For qualitative independent variables, odds ratios (OR) with $95 \%$ confidence intervals (CI) were obtained. Multivariate logistic regression was performed including all variables in order to exclude potential interacting confounding factors.

\section{Results}

A total of 104 cases and 208 age-matched controls were included in the study. A significant difference of 2 years in the age of menopause was detected (47.7 and 49.7 years, respectively, $p=0.01$ ), with a consistent mean increase in fertile life for the control group of 1.7 years ( 34.8 vs. 36.5 years, $p=0.004)$ and an estimated increase of $7 \%$ in the

Hormonal and Gynecological Risk Factors in FFA risk of suffering FFA for each year of advance in the age of menopause. Upon bivariate analysis (Table 1), significant differences between cases and controls were found in the frequency of hysterectomy (28.8 vs. $13.5 \%$, OR 2.14 [95\% CI 1.35-3.39], $p=0.002$ ), the frequency of breast cancer (7.7 vs. $2.4 \%$, OR 3.20 [95\% CI 1.07-9.54], $p=0.028$ ), use of an IUD ( 4.8 vs. $14.4 \%$, OR 0.34 [95\% CI 0.13-0.83], $p=$ 0.01 ), and use of tamoxifen (6.7 vs. $0.1 \%$, OR 7.00 [95\% CI $1.48-33.10], p=0.04$ ). After the multivariate analysis (Table 2), the only detected risk factor for FFA was previous intake of tamoxifen (OR 14.89 [95\% CI 2.42-91.68], $p=$ $0.004)$. The only protective factor was the previous use of an IUD (OR 0.22 [95\% CI 0.06-0.84], $p=0.027$ ).

Our results further support the previously suggested hypothesis of an underlying hormonal mechanism in the etiopathogenesis of FFA $[1,3,4]$. The potential role of estrogen as an antifibrotic and immunomodulatory agent in FFA has been discussed and may correlate with our findings [5]. Although the exact mechanism by which sexual hormones participate in the pathogenesis of FFA has yet to be elucidated, according to our results, situations that downregulate estrogenic function, such as menopause or treatment with tamoxifen, could play a role in the start or development of FFA as a result of loss of the downregulatory effect of estrogens over fibrosis and immunity.

\section{Limitations}

The main limitation of our study is a relatively low sample size. Because of the recall bias, patients were not able to accurately remember some dates, nor to differenti- 
Table 2. Final multivariate analysis

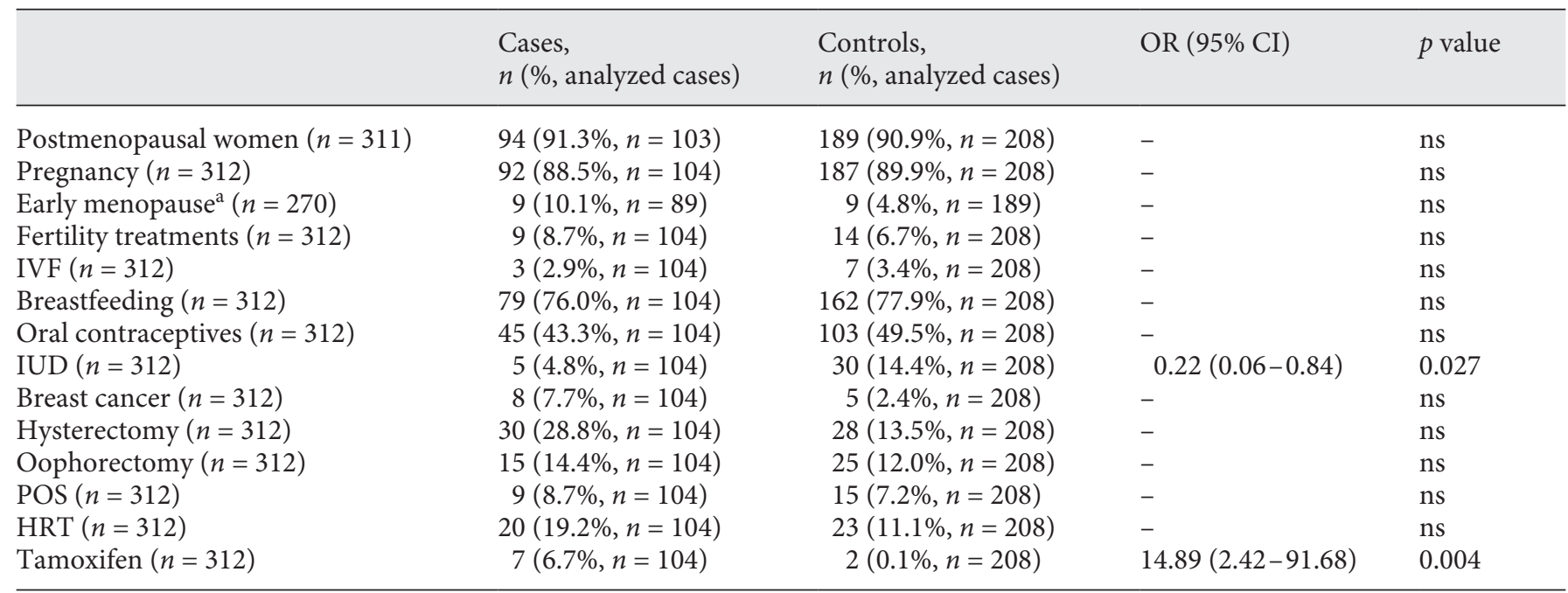

OR, odds ratio; CI, confidence interval; IVF, in vitro fecundation; IUD, intrauterine device; POS, polycystic ovary syndrome; HRT, hormone replacement therapy; ns, not significant. ${ }^{a}$ Menopause before age 40 years.

ate between the previous use of a cooper IUD or of a hormonal-releasing IUD. Body mass index or obesity were not recorded in our study and are potential confounding factors due to the hormonal function of fat tissue. The relationship of patients and controls with potential external hormonal disruptors was not assessed in this study.

\section{Conclusions}

In conclusion, an earlier age of menopause may imply a higher risk of developing FFA, and the use of an IUD as a contraceptive may protect against the development of FFA, but more studies are needed to confirm these associations and their implications. Intake of tamoxifen, with the induction of a low-estrogen environment around the hair follicle, may also trigger or maintain the pathogenic process of FFA.

Most certainly, there is no single cause for FFA, and this entity results from the dynamic interaction of genetic, environmental, and hormonal factors, each of them contributing in a variable percentage in every patient until a threshold for disease development is reached.

\section{Statement of Ethics}

Informed consent was obtained from all patients to participate in the study. The study protocol has been approved by the institutional committee on human research.

\section{Disclosure Statement}

The authors declare not having any conflicts of interest regarding this study. This work had no funding source.

\section{References}

1 Vañó-Galván S, Molina-Ruiz AM, SerranoFalcón C, Arias-Santiago S, Rodrigues-Barata AR, Garnacho-Saucedo G, Martorell-Calatayud A, Fernández-Crehuet $P$, Grimalt R, Aranegui B, Grillo E, Diaz-Ley B, Salido R, Pérez-Gala S, Serrano S, Moreno JC, Jaén P, Camacho FM: Frontal fibrosing alopecia: a multicenter review of 355 patients. J Am Acad Dermatol 2014;70:670-678.

2 Georgala S, Katoulis AC, Befon A, Danopoulou I, Georgala C: Treatment of postmenopausal frontal fibrosing alopecia with oral dutasteride. J Am Acad Dermatol 2009;61: 157-158.

3 Ladizinski B, Bazakas A, Selim MA, Olsen EA: Frontal fibrosing alopecia: a retrospective review of 19 patients seen at Duke University. J Am Acad Dermatol 2013;68:749-755.

4 Tosti A, Piraccini BM, Iorizzo M, Misciali C: Frontal fibrosing alopecia in postmenopausal women. J Am Acad Dermatol 2005;52:55-60.

5 Tziotzios C, Stefanato CM, Fenton DA, Simpson MA, McGrath JA: Frontal fibrosing alopecia: reflections and hypotheses on etiology and pathogenesis. Exp Dermatol 2016;25: 847-852. 